\title{
Role of Arthrodesis in Adult-acquired Flatfoot Deformity
}

\author{
Arthithat Kirinpanu
}

\begin{abstract}
Adult-acquired flatfoot deformity (AAFD) is composed of multi-structure problems. Failure of tendons, capsular, and other ligamentous structures lead to significant deformity and disability. Several therapeutic approaches are used to treat this disease. Arthrodesis reconstruction type procedure was raised as a satisfactory operation with high patient satisfaction. Ability to reduce most severe and fix deformities made it a procedure of choice in dealing with advance stage AAFD. Malunion, nonunion, lateral wound breakdown, and adjacent joint osteoarthritis are leading problems associated with this operation. However, there are some situations that patient will be best undergone these operations. Realigned triple arthrodesis along with its modifications are currently wide-accepted treatment for rigid arthritic flatfoot. Severe flexible AAFD and failed flatfoot reconstruction while choosing patients properly are also possible indications to treat with the fusion techniques. Lastly, some specific patient factors (age, morbid obesity, preexisting degenerative joint disease, and neuropathic patients) could be important factors to influence type of operations.
\end{abstract}

Keywords: Adult-acquired flatfoot deformity, Arthritis, Deformity, Double arthrodesis, Flatfoot, Fusion, Modified double arthrodesis, Posterior tibial tendon dysfunction (PTTD), Triple arthrodesis.

Journal of Foot and Ankle Surgery (Asia Pacific) (2021): 10.5005/jp-journals-10040-1140

\section{INTRODUCTION}

Adult-acquired flatfoot deformity (AAFD) is composed of multi-structure problems. Failure of tendons, capsular, and other ligamentous structures lead to significant deformity and disability. Typical deformity is characterized by flattening of the medial longitudinal arch, midfoot abduction, heel valgus, and forefoot varus. ${ }^{1}$ Severity can range from only medial ankle pain to progressive flexible deformity and eventually to fix deformity associated with difficulty in an ambulation.

Several therapeutic approaches are used to cope with this disease. In early stage, non-operative treatment seems to be the most appropriate way with the use of rehabilitation programs and some orthotic devices, such as University of California Berkeley Laboratory (UCBL). In later stage, operative treatments will be mandatory.

Flexible reconstruction procedures included medial calcaneal osteotomy (MDCO), lateral column lengthening $(\mathrm{LCL})$, posterior tibial tendon debridement or augmentation, tendon transfer, gastrosoleus complex lengthening, and others soft tissue procedures. This type of reconstructions has a pro in sparing joints thus preserve motions, flexibility, and less chance of adjacent osteoarthritis. Major drawbacks are inability to deal with severe deformities and higher rate of recurrence of deformities.

Arthrodesis reconstruction type procedure was initially introduced back by Hoke in $1921 .^{2}$ He performed a double arthrodesis of subtalar (ST) and talonavicular (TN) joints in paralytic feet. Triple arthrodesis of hindfoot was raised as a satisfactory operation with high patient satisfaction. ${ }^{3-5}$ Ability to reduce most severe and fix deformities made it a procedure of choice in dealing with advance stage AAFD. Complications with this procedure have been reported as much as its power. Malunion, nonunion, lateral wound breakdown, and adjacent joint osteoarthritis are leading problems with this operation. With those complications, many modifications for joints sacrificing operations are proposed to achieve good surgical outcomes and less complications. These include double arthrodesis [TN and calcaneocuboid (CC) fusion], modified double arthrodesis (TN and ST fusion), isolated ST or
Orthopedics Department, Phyathai Sriracha General Hospital, Chonburi, Thailand

Corresponding Author: Arthithat Kirinpanu, Orthopedics Department, Phyathai Sriracha General Hospital, Chonburi, Thailand, e-mail: arthithat@gmail.com

How to cite this article: Kirinpanu A. Role of Arthrodesis in Adultacquired Flatfoot Deformity. J Foot Ankle Surg (Asia Pacific) 2021;8(2):60-65.

Source of support: Nil

Conflict of interest: None

TN joint fusion, and other adjunctive soft tissue procedures. Accurate indications for each detailed procedure will ensure the best outcomes. This review article will focus on role of arthrodesis involved operations in AAFD (Fig. 1).

\section{Rigid Arthritic Aafd}

Rigid arthritic AAFD as stated by grade III of posterior tibial tendon dysfunction (PTTD) by Johnson and Strom is a deformity in which the malalignment at the triple joint complex (TN, CC, and ST joints) cannot be corrected past neutral with passive inversion. ${ }^{6,7}$ Deformity is fix so that negate the use of purely joint-sparing procedures. Most patients may have degenerative changes in the triple joint complex as a result of chronicity of malalignments.

Triple arthrodesis is the landmark procedure for this stage of disease. It can cope with both the massive malalignment and the arthritic changes in joint. ${ }^{3}$ The operation involves fusion of three joints-TN, CC, and ST joint. Traditional surgical exposure is a combined lateral and medial incision. ${ }^{8}$ Fusion operation begins with ST joint as it is reduced into neutral to slight valgus position $0^{\circ}$ to $5^{\circ}$. Varus hindfoot alignment is not acceptable because this will lock transverse tarsal joint and drive forefoot into varus position. Once hindfoot alignment is achieved, fixation is performed using 1 or 2 partially threaded cancellous screws of at least $6.5 \mathrm{~mm}$ in diameter. There are no consensus whether number, size, and direction of 

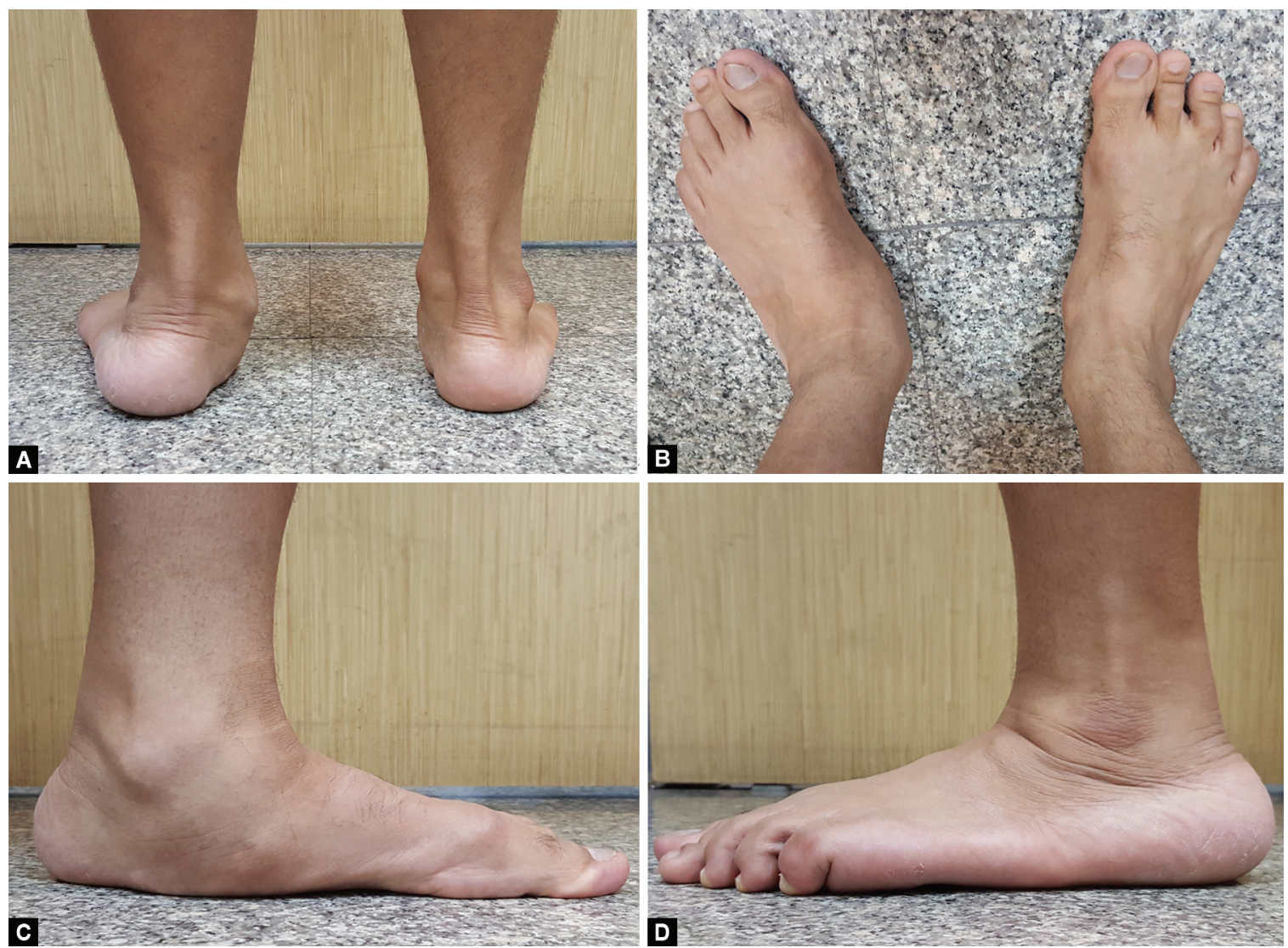

Figs 1 A to D: Typical deformities in AAFD patient (left side): (A) Heel valgus with too many toe signs; (B) Midfoot abduction; (C) Flattening of the medial longitudinal arch; (D) Impingement of lateral ankle

screws to use. ${ }^{9,10}$ Next step, TN joint should be reduced by inversion of midfoot and plantarflexion of first ray to reverse deformities. Either plate or compression screws is then used to fix the construct. Again, there are no consensus about fixations techniques. ${ }^{11}$ At last, CC joint should be examined for space. If it has a noticeable gap, then bone graft will have to be filled in order to avoid nonunion and shortening of fusion site.

Pell and colleagues ${ }^{12}$ reported medium-term follow-up study of 132 feet underwent triple arthrodesis to have a powerful ability of deformity correction, good satisfaction points (8.3/10), and acceptable postoperative modified American Orthopaedic Foot and Ankle Society (AOFAS) score (60.7/94). However, rate of adjacent ankle osteoarthritis had increased significantly, although patient satisfaction was not associated with the presence of arthritis. They concluded that triple arthrodesis is effective in relieving pain and decreasing functional deficits secondary to various etiologies and deformities. Czurda and associates in 2009 reported the similar outcomes in retrospective study of 20 patients. They found significant improvements in AOFAS score (average 51 points). Seventy-five percent of the patients had no or only slight pain. Nearly, all the patients were satisfied with the result of the surgery. ${ }^{13}$

Wound complications are the most common complication in early postoperative phase. Most of wound problems are from lateral incision because it is the tension side after corrections. In effort to reduce this problem, Jeng and colleague ${ }^{14}$ treated 17 patients who had a rigid hindfoot valgus deformity with triple fusion through a single medial incision. There were no wound complications but
CC fusion was not completed in two patients regarding exposure. Adequate CC joint exposure and difficulty in fixation could be limitation of this technique. Röhm and associates reported mid- to long-term results of 84 patients underwent TN and ST joint fusion through a medial incision (modified double arthrodesis). Most of the patients $(90.5 \%)$ can maintain the fusion construct until the last follow-up. Neither major wound complications nor need for further CC joint fusion was reported. ${ }^{15}$

In mid- to long-term postoperative phase, the problems will be shifted to limitations in an ambulation. Due to decreased overall motions, patients will have gait problems with walking inclines, accommodating uneven ground, and stair climbing ${ }^{16}$ Adjacent joint osteoarthritis is another major complication related to triple fusion. In some series, rate of osteoarthritis goes up high to nearly half of all patients. ${ }^{17,18}$ Increased stress to ankle and midfoot secondary to the stiffness from triple fusion construct is accounted for the accelerated wear of joints.

To deal with the late problem as osteoarthritis, many investigators shared an idea of fusing fewer joints to attain the deformity correction while preserving some hindfoot motions. In 1997, Astion and his colleagues conducted a cadaveric study about hindfoot motion after selective arthrodesis. They found that any combination of simulated arthrodesis that included the TN joint will lock the motion of the remaining joints to about $2^{\circ}$. As a result, they concluded that the TN joint is the key joint of the triple joint complex. The TN joint had the greatest range of motion, and simulated arthrodesis of this joint essentially eliminated motion of the other joints of the complex. ${ }^{19}$ 
Mann and Beaman reviewed 24 patients underwent double arthrodesis. The overall satisfaction rate was 83 and $76 \%$ of patients having good and excellent results. Adjacent osteoarthritis was observed in many patients, but most patients were asymptomatic. Nonunion of TN joint is the most complication in their study. ${ }^{20}$ Clain and Baxter also reported $75 \%$ of their patients (16 feet) to have excellent and good results following double arthrodesis operation. Progressive arthritis of the ankle was seen in six patients and of the naviculocuneiform joint in seven. ${ }^{21}$ These investigators recommended this procedure for patients with advance deformities in which arthritis focus on only transverse tarsal joint and a passively correctable non-arthritic ST joint. Thus, arthritis of ST joint should be compelling contraindication for this procedure.

Several surgeons found that it would be very hard to find the case without ST joint arthritis in such a later stage of rigid flatfoot. ${ }^{22}$ With this in mind, they recommended to include ST joint in fusion procedure. Moreover, they pointed out that CC should be left unfused. Sparing the CC joint has several advantages. It reduced the operative time and the risk of nonunion. ${ }^{23}$ Preserved CC joint provides some movement and did not further shorten lateral column length and thereby diminished the loading on adjacent joints which may lead to arthritis. ${ }^{12,19}$

Knupp et al. ${ }^{24}$ described a retrospective review of 32 feet underwent modified double fusion through a medial approach. Fusion was achieved in all feet at a mean of 13 weeks and no wound complications. Fusion construct remained stably unchanged at average 2-year follow-up time. Similar early term results were reported by Brilhault and Sammarco. Brilhault and colleagues stated that significant radiographic improvements were achieved in all of their patients while no report of major wound complications and an asymptomatic CC joint. ${ }^{25}$ Sammarco and his groups showed a marked AOFAS Ankle-Hindfoot Scale improvement in the study 44.7 preoperatively to 77.0 postoperatively $(p<0.01)$. Moreover, their patients experienced improvements in pain, function, cosmetic, and shoe wear. ${ }^{23}$ To date, there are no studies that report long-term outcomes with this selective fusion procedure. Longer follow-up times may need to prove the better results of this technique.

Recently, some studies were established to compare the results between traditional triple arthrodesis and modified double arthrodesis. DeVries and Scharer conducted a retrospective radiographic to compare these operations (40 cases, each 20 consecutives cases). Both groups demonstrated ability to reduce severe deformities and postoperative results were similar. ${ }^{26}$ In contrast, Burrus and his associates reviewed medical record of patients who had undergone either a modified double $(n=9)$ or triple $(n=7)$ arthrodesis for stage 3 flatfoot deformity. Interestingly, modified double fusion group had significant lower mean Foot and Ankle Ability Measure ADL score. All of triple arthrodesis patient achieved satisfactory union but modified double arthrodesis group had a union rate only $56 \%$. The main limitation of this study was small number subjects. More population number will ensure the results. ${ }^{27}$ In 2020, the American College of Foot and Ankle Surgeons (ACFAS) had a consensus statement regarding TN and ST joint fusion in combination or in isolation for effective treating rigid flatfoot deformity. They concluded that this statement is neither appropriate nor inappropriate and decision to fuse which joints should be based on symptoms and magnitudes of deformity corrections (Fig. 2). ${ }^{28}$

\section{Severe Flexible AAFD}

Joint preserving procedure is currently in trend with treatment of stage 2 flexible flatfoot. Many combinations of bony and softtissue procedures were applied to get the best outcomes rather than just one isolated procedure. However, some surgeons advocated limited fusion techniques as their primary treatment. In hope of stable and reliable results, Cohen and Johnson proposed realignment ST arthrodesis as an effective treatment of the planovalgus deformity of posterior tibial tendon insufficiency. Union rates from their series was approaching $100 \% .{ }^{29}$ Johnson et al. recommended ST arthrodesis combined with spring ligament repair/reefing and flexor digitorum longus (FDL) transfer to the navicular to treat stage PTTD. Indication for their operation was the presence of a flexible planovalgus deformity without fixed forefoot varus or degenerative changes at the transverse tarsal joints. They believed that direct correction of ST joint was more predictable and durable than indirect correction through changing of musculotendinous pulled line axis (such as calcaneus osteotomy, LCL). Second, addition of the medial soft tissue reconstruction, including tendon transfer and TN capsule/spring ligament complex imbrication, provided additional correction of forefoot abduction and midfoot pronation, restored plantar flexor power at the ankle, and reduced the abnormal valgus alignment. The study results showed favorably outcomes as good as with flexor transfer combined with either calcaneal osteotomy or LCL. ${ }^{30}$

Severely compromised spring ligament could be another recommendation for selective fusion. Sometimes in severe case, there was not enough tissue for reconstruction or not strong enough for constructs. Some surgeons gave an idea of limited fusion instead of ligament reconstruction for better reliable alignment. ${ }^{31}$ Despite more predictable outcomes in severe flexible flatfoot patients, many investigators still aware of complications like nonunion. Latest study in 2018 was conducted to compare reoperation rates and complication rates following flexible jointsparing reconstruction vs fusion procedures in flexible AAFD patients. Nonunion rate was higher in fusion group (16\%), while flexible reconstruction group was only $4 \%$. However, rates of reoperation for nonunion were similar between groups. This would reflect that most nonunion might be asymptomatic. ${ }^{32}$

\section{Failed Flatfoot Reconstruction}

While dealing with the case of previous failed operative treatment, the important things are to address the cause of failure and recognize what is exactly patient's problems. Overall alignment should always be examined. If alignment is acceptable, it means that problem would be from nonunion sites or arthritic joints. Regarding degenerative joint disease, arthrodesis would have a role in this problem. Selective fusion of involved joint would be a solution. However, decision to fuse which joint should be based on severity, location of pain, and other patient factors (age, demand of patient, underlying). In the other cause, nonunion should be repaired with or without bone graft. ${ }^{33}$

If malalignment is presented, every components should be checked. Forefoot, midfoot, and hindfoot including ankle or in combinations could lead to a potential threat. Depending on location shape of deformities and what was done prior, decisions can then be made regarding corrective osteotomy vs realignment fusion. 

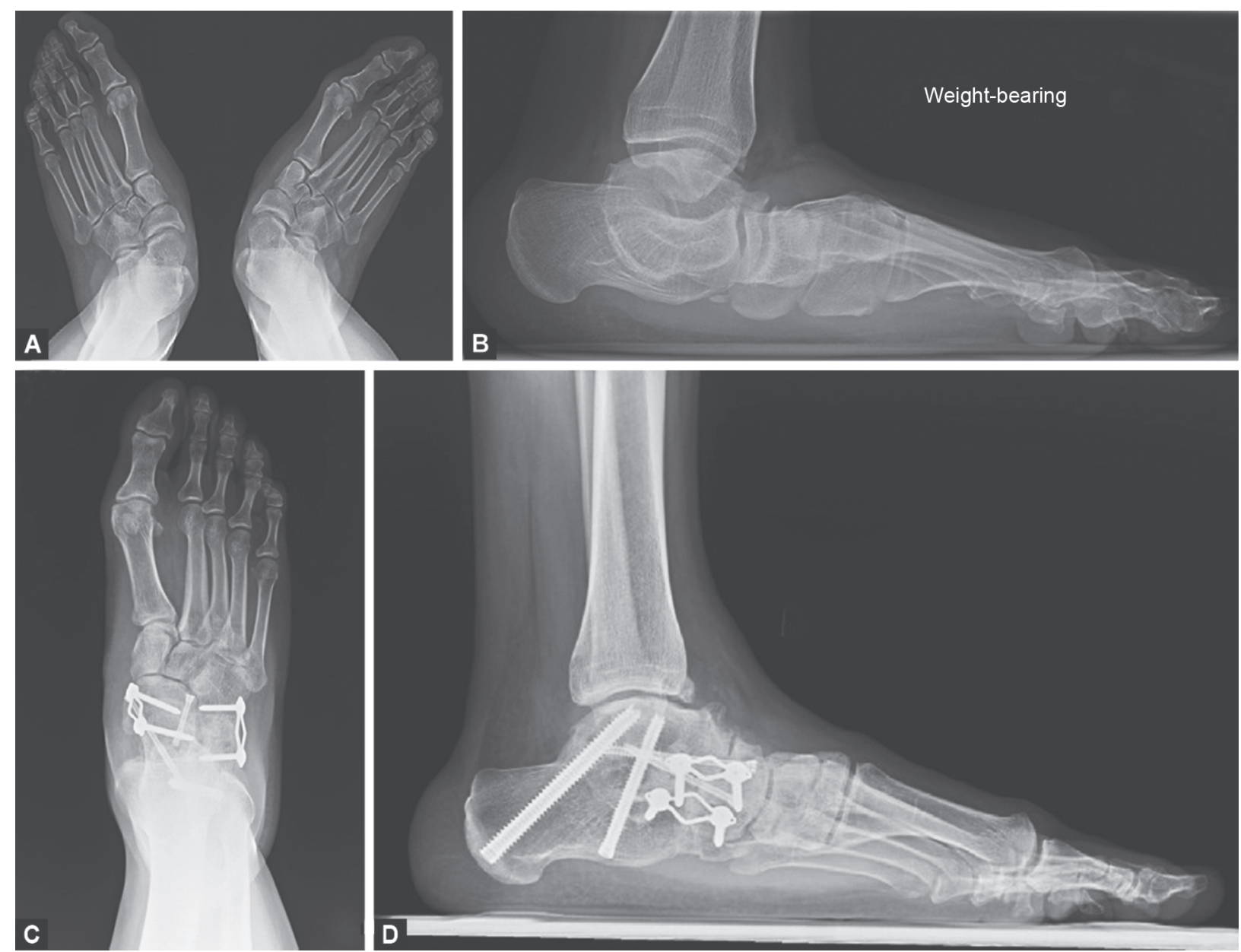

Figs 2A to D: (A and B) Preoperative radiographs of a male patient with severe AAFD most on right side. Hyperabduction midfoot and severe arch collapsed were noted; (C and D) After treatment with triple fusion procedure, postoperative radiographs showed nearly anatomic alignment foot. Pictures from Shawen et al. ${ }^{34}$

Malalignment could be categorized into two typesundercorrection and overcorrection. About undercorrected group, the usual deformities are forefoot varus, midfoot abduction, and hindfoot valgus liked untreated flatfoot. Surgical options treating residual hindfoot valgus included medializing calcaneal osteotomy, ST arthroereisis, and ST arthrodesis. Hindfoot arthritis and its flexibility are factors to dictate choice of procedure. ${ }^{35}$

Residual midfoot abduction is corrected by LCL procedures. Both calcaneal opening wedge osteotomy (Evans) and CC distraction arthrodesis have the ability to accomplish this. ${ }^{35}$ Controversy still arise inappropriate indication for these procedures. Thomas et al. reported result in 27 feet underwent LCL procedures by comparing these techniques. Both operations showed significant improvements in radiographic parameters and postoperative AOFAS scores. But there was a concern about high rate of nonunion and delayed union associated with the CC distraction arthrodesis group. ${ }^{36}$

Focus on residual forefoot varus, first tarsometatarsal (TMT) arthrodesis and medial cuneiform opening wedge osteotomy (cotton osteotomy) are among top-notch procedures. Tarsometatarsal complex hypermobility is the key to select between these two procedures. ${ }^{35}$ "Plantar gap sign" (Fig. 3) as seen on lateral weightbearing foot radiograph would suggest midfoot instability (hypermobility). If we could detect this situation or arthritis of

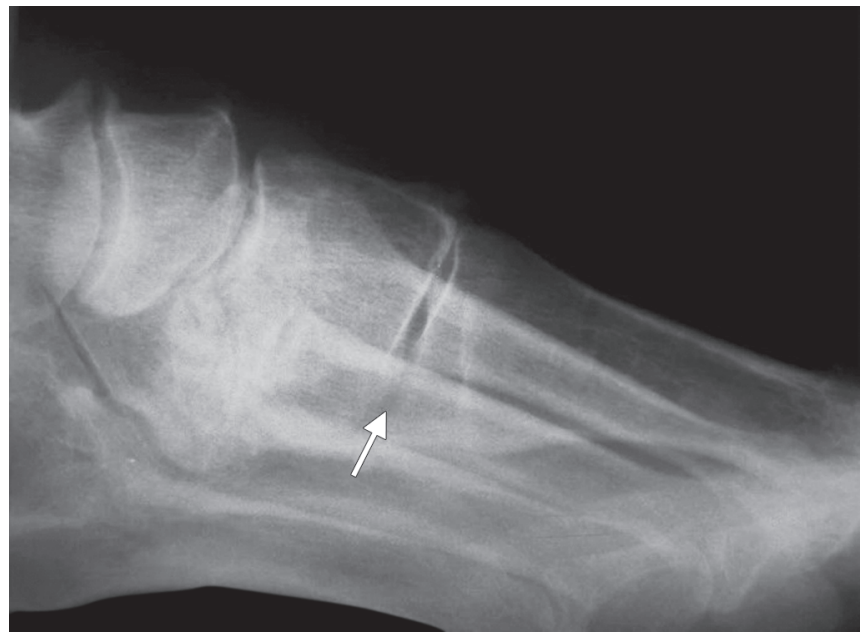

Fig. 3: "Plantar gap sign" (arrow) as seen from lateral weight-bearing radiograph could be reflected of midfoot hypermobility. Picture from Popelka et al. ${ }^{37}$

midfoot, TMT fusion procedure is then more suitable operation. Although TMT arthrodesis had a bad reputation of high rate of nonunion (10-12\%), some studies provided promising results. 
Thompson and his colleagues conducted a retrospective study in 182 patients underwent modified Lapidus procedure or tarsometatarsal joint (TMTJ) arthrodesis as part of a flatfoot reconstruction. Their nonunion rate was as low as 4 and $2 \%$ revision rate. They believed that meticulous operative technique, proper prepared fusion site, rigid internal fixation, using local bone grafting, tendoachilles lengthening or gastrocnemius slides for equinus contractures, and postoperative immobilization are the key for their success. ${ }^{38}$

Minority of failed foot reconstruction case had an overcorrected alignment. The deformities typical look like a cavovarus foot. Every components of foot had to be analyzed as in the treatment of undercorrected cases. ${ }^{33}$ Most soft tissue problems, such as an overlengthening of the gastrosoleus complex, overtightening of FDL tendon transfer, could be solved by physical therapy. Hindfoot varus from excessive inferomedial translation usually well treated with various techniques of calcaneal osteotomy. ${ }^{39}$

Lateral foot discomfort is the chief complaint of overlengthening lateral column. Then, shortening lateral column procedures either through closing wedge osteotomy or shortening arthrodesis should be considered. Lastly, excessive plantar flexed first ray from overcorrection medial column could pose a pain around first ball and sesamoids area. Dorsiflexion closing wedge osteotomy of midfoot would have a role for this condition. Otherwise, if the medial joint complex had an arthritis, realignment arthrodesis (dorsal closing wedge or superior translation) would be more appropriated. ${ }^{39}$

\section{Specific Patient Factors}

Several investigators believed that some patient's factors would be a benefit from joint fusion procedure more than the others. Morbid obesity is one of the first factors to be concern. More weight means more load to reconstruction construct. Better stable construction would maintain correction of the deformity. A study by Nielsen et al. shown a worse outcomes with obesity [body mass index $(\mathrm{BMI})>30$ ] but failed to make it statistical significant. ${ }^{40}$ Age is also an importance factor. Older patients always get along with comorbidities and sedentary lifestyles. Thus, these patients should undergo just only one definite surgery. Arthrodesis is a more reliable procedure and its short-term outcome is equally good as other operation. ${ }^{31}$ Preexisting degenerative disease from past injuries or other conditions should be better off with selective fusion in involving joints as joint-sparing procedure is unlikely to solve the problem. Lastly, patients with neuropathic deformity, such as Charcot arthropathy, should be strongly recommended to undergo arthrodesis type operation. These patients lose their protective sensation to protect themselves. Strong and durable reconstruction will be a preferable operation. ${ }^{34}$

\section{Conclusion}

Adult-acquired flatfoot deformity had a wide spectrum of disease. The symptoms range from minor pain with flexible deformity to fixed painful multiple directions deformities and arthritis. Thus, various treatments are proposed in order to get the best outcomes. Arthrodesis procedure and its variants have good reputations in sustained predictable results with irresistible short- to mid-term outcomes. In spite of this, their late major drawbacks including limitation in an ambulation due to joint stiffness and adjacent osteoarthritis make this procedure to be used as a salvaged operation. Every attempts should go toward joint-sparing procedures before proceed to fusion operations. However, arthrodesis procedures still play a main role in treating rigid arthritic flatfoot patients. Other possible indications for this operation are severe flexible flatfoot deformity, failed flatfoot reconstruction, and some specific patient factors (age, morbid obesity, preexisting degenerative joint disease, and neuropathic patients).

\section{References}

1. Vulcano E, Deland JT, Ellis SJ. Approach and treatment of the adult acquired flatfoot deformity. Curr Rev Musculoskelet Med 2013;6(4):294-303. DOI: 10.1007/s12178-013-9173-z.

2. Hoke M. An operation for stabilizing paralytic feet. JBJS 1921;3(10): 494-507.

3. Bennett GL, Graham CE, Mauldin DM. Triple arthrodesis in adults. Foot Ankle 1991;12(3):138-143. DOI: 10.1177/107110079101200302.

4. Angus $P$, Cowell $H$. Triple arthrodesis. A critical long-term review. J Bone Joint Surg Br 1986;68(2):260-265. DOI: 10.1302/0301620X.68B2.3958012.

5. Fortin PT, Walling AK. Triple arthrodesis. Clin Orthop Relat Res 1999;365:91-99. DOI: 10.1097/00003086-199908000-00012.

6. Johnson KA, Strom DE. Tibialis posterior tendon dysfunction. Clin Orthop Relat Res 1989;239(239):196-206. DOI: 10.1097/00003086198902000-00022.

7. Deland JT. Adult-acquired flatfoot deformity. J Am Acad Orthopae Surg 2008;16(7):399-406. DOI: 10.5435/00124635-200807000-00005.

8. Johnson J, Yu J. Arthrodesis techniques in the management of stage II and III acquired adult flatfoot deformity. Instr Course Lect 2006;55:531.

9. Gosch C, Verrette R, Lindsey DP, et al. Comparison of initial compression force across the subtalar joint by two different screw fixation techniques. J Foot Ankle Surg 2006;45(3):168-173. DOI: 10.1053/j.jfas.2006.02.001.

10. Lee JY, Lee YS. Optimal double screw configuration for subtalar arthrodesis: a finite element analysis. Knee Surg Sports Traumatol Arthrosc 2011;19(5):842-849. DOI: 10.1007/s00167-010-1383-y.

11. Jarrell SE, Owen JR, Wayne JS, et al. Biomechanical comparison of screw versus plate/screw construct for talonavicular fusion. Foot Ankle Int 2009;30(2):150-156. DOI: 10.3113/FAI-2009-0150.

12. Pell IVRF, Myerson MS, Schon LC. Clinical outcome after primary triple arthrodesis. JBJS 2000;82(1):47-57. DOI: 10.2106/00004623200001000-00006.

13. Czurda T, Seidl M, Seiser A, et al. Triple arthrodesis in treatment of degenerative hindfoot deformities: Clinical, radiological and pedobarographic results. Z Orthop Unfall 2009;147(3):356-361. DOI: 10.1055/s-0029-1185407.

14. Jeng $\mathrm{CL}$, Vora $\mathrm{AM}$, Myerson MS. The medial approach to triple arthrodesis. Indications and technique for management of rigid valgus deformities in high-risk patients. Foot Ankle Clin 2005;10(3):515-521. DOI: 10.1016/j.fcl.2005.04.004.

15. Röhm J, Zwicky L, Horn Lang T, et al. Mid-to long-term outcome of 96 corrective hindfoot fusions in 84 patients with rigid flatfoot deformity. Bone Joint J 2015;97(5):668-674.

16. Wu W-L, Huang P-J, Lin C-J, et al. Lower extremity kinematics and kinetics during level walking and stair climbing in subjects with triple arthrodesis or subtalar fusion. Gait Posture 2005;21(3):263-270. DOI: 10.1016/j.gaitpost.2004.02.001.

17. Klerken T, Kosse NM, Aarts CA, et al. Long-term results after triple arthrodesis: influence of alignment on ankle osteoarthritis and clinical outcome. Foot Ankle Surg 2019;25(2):247-250. DOI: 10.1016/j. fas.2017.11.003.

18. de Groot IB, Reijman M, Luning HA, et al. Long-term results after a triple arthrodesis of the hindfoot: function and satisfaction in 36 patients. Int Orthop 2008;32(2):237-241. DOI: 10.1007/s00264-006-0295-4.

19. Astion DJ, Deland JT, Otis JC, et al. Motion of the hindfoot after simulated arthrodesis. JBJS 1997;79(2):241-246. DOI: 10.2106/00004623-199702000-00012. 
20. Mann RA, Beaman DN. Double arthrodesis in the adult. Clin Orthopaed Relat Res 1999;365:74-80. DOI: 10.1097/00003086199908000-00010.

21. Clain MR, Baxter DE. Simultaneous calcaneocuboid and talonavicular fusion. Long-term follow-up study. J Bone Joint Surg Br 1994;76(1): 133-136. DOI: 10.1302/0301-620X.76B1.8300657.

22. Francisco R, Chiodo CP, Wilson MG. Management of the rigid adult acquired flatfoot deformity. Foot Ankle Clin 2007;12(2):317-327. DOI: 10.1016/j.fcl.2007.03.013.

23. Sammarco VJ, Magur EG, Sammarco GJ, et al. Arthrodesis of the subtalar and talonavicular joints for correction of symptomatic hindfoot malalignment. Foot Ankle Int 2006;27(9):661-666. DOI: 10.1177/107110070602700901.

24. Knupp M, Schuh R, Stufkens S, et al. Subtalar and talonavicular arthrodesis through a single medial approach for the correction of severe planovalgus deformity. J Bone Joint Surg Br 2009;91(5): 612-615. DOI: 10.1302/0301-620X.91B5.21727.

25. Brilhault J. Single medial approach to modified double arthrodesis in rigid flatfoot with lateral deficient skin. Foot Ankle Int 2009;30(1): 21-26. DOI: 10.3113/FAI.2009.0021.

26. DeVries JG, Scharer B. Hindfoot deformity corrected with double versus triple arthrodesis: radiographic comparison. J Foot Ankle Surg. 2015;54(3):424-427.

27. Burrus MT, Werner BC, Carr JB, et al. Increased failure rate of modified double arthrodesis compared with triple arthrodesis for rigid pes planovalgus. J Foot Ankle Surg 2016;55(6):1169-1174.

28. Piraino JA, Theodoulou, et al. American college of foot and ankle surgeons clinical consensus statement: appropriate clinical management of adult-acquired flatfoot deformity. J Foot Ankle Surg 2020;59(2):347-355.

29. Cohen BE, Johnson JE. Subtalar arthrodesis for treatment of posterior tibial tendon insufficiency. Foot Ankle Clin 2001;6(1):121-128. DOI: 10.1016/S1083-7515(03)00085-8
30. Johnson JE, Cohen BE, DiGiovanni BF, et al. Subtalar arthrodesis with flexor digitorum longus transfer and spring ligament repair for treatment of posterior tibial tendon insufficiency. Foot Ankle Int 2000;21(9):722-729. DOI: 10.1177/107110070002100902.

31. Henry JK, Shakked R, Ellis SJ. Adult-acquired flatfoot deformity. Foot Ankle Orthopaed 2019;4(1):2473011418820847. DOI: $10.1177 / 2473011418820847$.

32. Whitelaw K, Shah S, Hagemeijer N, et al. Fusion versus flexible reconstruction for patients with flexible flatfoot. Foot Ankle Orthopaed 2018;3(3):2473011418S00516.

33. Irwin TA. Revision of the failed flatfoot. Revision surgery of the foot and ankle, Surg Strateg Techniq 2019. p. 259.

34. Shawen SB, Dworak TC. Severe stage 2: fuse or reconstruct. Foot and Ankle Clinics 2017;22(3):637-642.

35. Hunt KJ, Farmer RP. The undercorrected flatfoot reconstruction. Foot and Ankle Clin 2017;22(3):613-624.

36. Thomas RL, Wells BC, Garrison RL, et al. Preliminary results comparing two methods of lateral column lengthening. Foot Ankle Int 2001;22(2):107-119. DOI: 10.1177/107110070102200205.

37. Popelka S, Hromádka R, Vavř́k P, et al. Hypermobility of the first metatarsal bone in patients with rheumatoid arthritis treated by lapidus procedure. BMC Musculoskelet Disord 2012;13(1):148. DOI: 10.1186/1471-2474-13-148

38. Thompson IM, Bohay DR, Anderson JG. Fusion rate of first tarsometatarsal arthrodesis in the modified Lapidus procedure and flatfoot reconstruction. Foot Ankle Int 2005;26(9):698-703. DOI: $10.1177 / 107110070502600906$

39. Irwin TA. Overcorrected flatfoot reconstruction. Foot Ankle Clin 2017;22(3):597-611.

40. Nielsen MD, Dodson EE, Shadrick DL, et al. Nonoperative care for the treatment of adult-acquired flatfoot deformity. J Foot Ankle Surg 2011;50(3):311-314. DOI: 10.1053/j.jfas.2011.02.002. 